\title{
Does GPAQ exhibit a higher correlation with the maximum oxygen capacity than IPAQ among young adults?
}

\author{
Gilang Fachri Maulana ${ }^{\text {abcde }} \mathbb{( 1 )}$, Novita Intan Arovah Chandra ${ }^{\text {abde } *(\mathbb{C})}$ \\ Universitas Negeri Yogyakarta, Indonesia
}

Received: 25 July 2021; Accepted 17 September 2021; Published 15 December 2021

Ed 2021; 6(3): 411-420

\begin{abstract}
International Physical Activity Questionnaire (IPAQ) and the Global Physical Activity Questionnaire (GPAQ) are the two most widely used to measure physical activity (PA). Yet, their convergent validation with the maximum oxygen capacity $\left(\mathrm{VO}_{2} \mathrm{Max}\right)$ among Indonesian young adults is unknown. This study compared physical activity (PA) assessments using IPAQ and GPAQ and their correlations with the maximal oxygen capacity $\left(\mathrm{VO}_{2} \mathrm{Max}\right)$ in the young adult population. This study was a cross-sectional descriptive study on 63 Sports Science students (84.13\% Male age ranged $18-22$ years). PA was measured using both the GPAQ and IPAQ to calculate the total PA and PA in each domain (i.e. work-related, transport-related, and leisure). One additional domain which was household-related was assessed exclusively for the IPAQ based on the scoring guideline. The $\mathrm{VO}_{2} \mathrm{Max}$ was measured using a standard equation based on participants' performance on a 1-mile run. Paired sample t-test was conducted to compare total PA based on GPAQ and IPAQ while the Wilcoxon sign rank test was conducted to compare GPAQ and IPAQ domains. Pearson or Spearman correlation test assessed the correlations among the $\mathrm{VO}_{2} \mathrm{Max}$, total PA, and PA on each GPAQ's and IPAQ domain. No significant difference in the total PA per week between IPAQ and GPAQ was found. Significant differences, however, were found in work and transport domains, but not in the leisure domains. A moderate correlation among GPAQ and IPAQ $(\mathrm{r}=0.56, p=0.01)$, a moderate correlation between GPAQ and $\mathrm{VO}_{2} \mathrm{Max}(\mathrm{r}=0.41, p=$ $0.01)$ but a weak correlation between IPAQ dan $\mathrm{VO}_{2} \mathrm{Max}(\mathrm{r}=0.25, p<0.05)$ were demonstrated. While, this study has indicated convergent validity of both GPAQ and IPAQ, GPAQ has a higher correlation with $\mathrm{VO}_{2} \mathrm{Max}$ compared to the IPAQ. Therefore, GPAQ is more recommended to be used to measure PA levels among Indonesian young adults compared to IPAQ.
\end{abstract}

Keywords: IPAQ; GPAQ; maximum oxygen capacity

https://doi.org/10.25299/sportarea.2021.vol6(3).7589

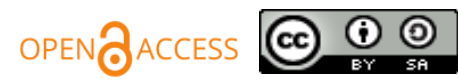

Copyright @ 2021 Gilang Fachri Maulana, Novita Intan Arovah Chandra

Corresponding Author: Gilang Fachri Maulana, Postgraduate Program of Sport Science, Universitas Negeri Yogyakarta, Yogyakarta, Indonesia

Email: gilang0123pasca.2019@student.uny.ac.id

How to Cite: Maulana, G. F., \& Chandra, N. I. A. (2021). Does GPAQ exhibit a higher correlation with the maximum oxygen capacity than IPAQ among young adults?. Journal Sport Area, 6(3), 411-420. https://doi.org/10.25299/sportarea. 2021.vol6(3).7589

Authors' Contribution: a - Study Design; b - Data Collection; c - Statistical Analysis; d - Manuscript Preparation; e - Funds Collection

\section{INTRODUCTION}

Physical activity is important for maintaining and improving health (Rhodes, Janssen, Bredin, Warburton, \& Bauman, 2017). The WHO recommends that adults get at least 150-300 minutes of moderate-intensity 
aerobic exercise, or at least 75-150 minutes of high-intensity aerobic exercise (Bull et al., 2020). Recommendations from the American Heart Association (AHA) and American College of Sports Medicine (ACSM) state that all healthy adults ages 18 to 65 should get at least 30 minutes of moderate-intensity aerobic exercise or at least 20 vigorous-intensity aerobic exercise every week. At least three days a week of a combination of the two (Haskell et al., 2007). Some tools such as pedometers and accelerometers have been used to measure physical activity (Cain, Sallis, Conway, Van Dyck, \& Calhoon, 2013; Herrmann, Heumann, Der Ananian, \& Ainsworth, 2013; Raustorp \& Fröberg, 2018). However, this method is relatively more expensive and cannot be used for certain physical activities (Cain et al., 2013). Several self-report questionnaires have been developed to measure physical activity. These instruments are used more frequently in public health research, because of the ease of implementation, low burden, low cost, and valuable information (Ács et al., 2020). The two most widely used questionnaires for evaluating physical activity worldwide are the International Physical Activity Questionaire (IPAQ) and Global Physical Activity Questionaire (GPAQ).

IPAQ was developed to measure physical activity at the population level and had been translated and validated in 12 countries (Craig et al., 2003). There are two versions of IPAQ, namely the long format and the short format. The long-form IPAQ assesses the four areas of physical activity (occupation, transportation, family, and leisure) (Craig et al., 2003). The long version of the IPAQ provides comprehensive and detailed information about daily physical activity habits in 4 different fields. These fields include leisure time, occupation, housework, and transportation activities (Hallal et al., 2010). The long format IPAQ is also utilized in large-scale sports activity research to achieve detailed information about the intensity, frequency, and location of the reported activity (Stelmach, 2018). The short version of th IPAQ includes items on walking, moderate and vigorous physical activity, and sedentary behavior (Hallal et al., 2010). The development of the questionnaire is applicable to an extensive range of languages and cultures (Stelmach, 2018). The leisure and transportation is recommended to use as the part of the long format IPAQ to do monitoring and research to record the level of physical activity in Latin America (Hallal et al., 2010).

GPAQ was developed by the WHO and used for physical monitoring in various countries. It has been translated and verified in 9 countries/regions (Armstrong \& Bull, 2006). The main result variables of the GPAQ analysis are categorical variables of total physical activity (high, medium, low); continuous variables of total physical activity in each fieldwork, transport, leisure (reported as median METmin/week) (Armstrong \& Bull, 2006). GPAQ was produced for face-to-face interviews managed by employees trained to confront difficulties such as reading problems (Wanner et al., 2017). GPAQ is designed to monitor research in developing countries. Its basic principles and structures make it possible to measure various physical activities that improve health in multiple countries (Soo, Manan, \& Suriati, 2015)a. GPAQ was also developed as an intermediate tool between the two versions of IPAQ to minimize differences in physical activity assessments between different countries (Stelmach, 2018).

Cardiovascular health seems to play a role in the accuracy of self-reported moderate and vigorous PA (Taylor, Tomaz, Lambert, \& Karpul, 2016). Both GPAQ and IPAQ have been used to predict maximum oxygen uptake from adolescents to adults (Kamyan, Labania, Kamyan, Rahman, \& Bagchi, 2020; Nainggolan, Indrawati, \& Pradono, 2018; Piccinno \& Colella, 2017). However, few studies have indicated some discrepancies with those findings. To illustrate, a study by Nainggolan et al., (2018) reported that GPAQ was not correlated with $\mathrm{VO}_{2} \mathrm{Max}(\mathrm{r}=0 ., 070$ and $\mathrm{P}$ value=0.44), while IPAQ was found to be unrelated to maximal oxygen uptake (Bahnemiri, Roshan, \& Movaghar, 2018). Further research, therefore is required to confirm these findings. Moreover, those studies have assessed the correlation between IPAQ and GPAQ with $\mathrm{VO}_{2} \mathrm{Max}$ separately. Thus, comparison between GPAQ and IPAQ could not be made. They were also conducted in general population, thus it is unknown wether the correlation between GPAQ and IPAQ with $\mathrm{VO}_{2} \mathrm{Max}$ is applied in the specific population such as young adults who tend to be physically active such as Sports Science students in the Indonesian context. Therefore, this study aimed to compare the assessment of physical activity between IPAQ and GPAQ and their correlations with the maximum oxygen capacity $\left(\mathrm{VO}_{2} \mathrm{Max}\right)$ in this population. This study was expected to provide information and justify the use of the instruments in this target population. 


\section{METHOD}

This study was a cross-sectional descriptive study conducted in the Physical, Health, and Recreation Education study program at Sports Science Faculty, Yogyakarta State University in September 2019.

\section{Assessment of Physical Activity Levels}

Assessments of physical activity were using GPAQ and IPAQ. The GPAQ consists of 16 questions which probed 3 physical activity domains, namely work-related, transport, and leisure-based physical activity domains. It also measures sedentary activity. GPAQ scoring protocol (WHO, 2012) was used to calculate the total METs score from GPAQ. The IPAQ consists of 27 questions that assess 4 domains which were the same domains with GPAQ with an additional domain (i.e. household-related physical activity domain). It also assesses time spent in sedentary activity. IPAQ scoring protocol (IPAQ Research Committee, 2005) was used to calculate the total METs score from IPAQ. Both the GPAQ and IPAQ were translated into the Indonesian language.

\section{Assessment of Maximum Oxygen Capacity}

Measurement of maximum oxygen capacity $\left(\mathrm{VO}_{2} \mathrm{Max}\right)$ was done by doing a 1-mile run. Participants were instructed to complete the one-mile distance as quickly as possible. If the participant cannot continue running, walking is allowed. The test was carried out on a $400 \mathrm{~m}$ track. Calculation of $\mathrm{VO}_{2} \mathrm{Max}$ was based on age, gender, and BMI using an equation from Cureton (Cureton, Sloniger, O'Bannon, Black, \& McCormack, 1995). $\mathrm{VO}_{2} \mathrm{Max}=0.21$ (agexgender)-0.84 (BMI)-8.41 (time)+0.34 (time $\left.{ }^{2}\right)+108.94$. Where gender $=1$ for male; 0 for female; time $=$ minutes.

\section{Anthropometric and Social Demography Measures}

Data for anthropometry were weight in kilograms and height in meters. Data on weight and height were used to calculate Body Mass Index (BMI). Body Mass Index (BMI) was calculated as body mass in kilograms divided by height in squared meters $(\mathrm{kg} / \mathrm{m} 2)$. Sociodemographic data included age and gender.

\section{Statistical Analysis}

Paired sample t-test was conducted to compare GPAQ and IPAQ. Wilcoxon sign rank test was conducted to see the differences between GPAQ and IPAQ domains. Pearson and Spearman correlation tests were carried out on the $\mathrm{VO}_{2} \mathrm{Max}$ variable, GPAQ total score, GPAQ domains, IPAQ total score, and IPAQ domains to determine the significance of the correlation among them. The analysis was performed using SPSS version 25 .

\section{RESULTS AND DISCUSSION}

A total sample of 63 people ( $84.13 \%$ were male) aged 18 to 23 years (mean $=19.6 \pm 0.7)$ participated in this study. They included 53 males $(84.1 \%)$ and 10 females $(15.9 \%)$ and were on average considered normal weight. Table 1 shows descriptive statistics of the sample in total participants and based on sex.

Table 1. Descriptive Statistic of the Sample

\begin{tabular}{cccc} 
& $\begin{array}{c}\text { Total }(\mathbf{n}=\mathbf{6 3}) \\
\text { Mean } \pm \text { SD }\end{array}$ & $\begin{array}{c}\text { Male }(\mathbf{n}=\mathbf{5 3}) \\
\text { Mean } \pm \text { SD }\end{array}$ & $\begin{array}{c}\text { Female }(\mathbf{n}=\mathbf{1 0}) \\
\text { Mean } \pm \text { SD }\end{array}$ \\
\hline Age & $19.6 \pm 0.7$ & $19.6+0.6$ & $19.9+1.2$ \\
Weight & $59.5 \pm 10.6$ & $60.7+10.7$ & $53.1+7.3$ \\
Height & $167.1 \pm 7.3$ & $168.6+6.1$ & $158.9+7.7$ \\
BMI & $21.2 \pm 2.7$ & $21.3+2.9$ & $20.9+1.3$ \\
\hline
\end{tabular}

Table 2 shows descriptive statistics and $\mathrm{VO}_{2} \mathrm{Max}$ in the gender group. All physical activity and domains scores were higher in the male than female group, as well as $\mathrm{VO}_{2} \mathrm{Max}$ scores. But, males and females have the same score in sedentary domain of GPAQ. Female have higher score in domestic domain. 
Table 2. Descriptive Statistic of VO2Max, GPAQ and IPAQ

\begin{tabular}{|c|c|c|c|}
\hline & $\begin{array}{c}\text { Total }(n=63) \\
\text { Mean } \pm \text { SD }\end{array}$ & $\begin{array}{c}\text { Male }(n=53) \\
\text { Mean } \pm \text { SD }\end{array}$ & $\begin{array}{c}\text { Female }(n=10) \\
\text { Mean } \pm \text { SD }\end{array}$ \\
\hline \multicolumn{4}{|l|}{ GPAQ } \\
\hline Total & $10737 \pm 4980$ & $11242 \pm 4807$ & $8058 \pm 5277$ \\
\hline Work & $5262 \pm 2774$ & $5485 \pm 2645$ & $4080 \pm 3275$ \\
\hline Transport & $1057 \pm 2630$ & $1178 \pm 2842$ & $414 \pm 661$ \\
\hline Leisure & $4418 \pm 2391$ & $4580 \pm 2426$ & $3564 \pm 2100$ \\
\hline Sedentary & $262 \pm 124$ & $262 \pm 132$ & $262 \pm 75$ \\
\hline \multicolumn{4}{|l|}{ IPAQ } \\
\hline Total & $10724 \pm 6826$ & $11256 \pm 7104$ & $7902 \pm 4342$ \\
\hline Work & $5266 \pm 5201$ & $5523 \pm 5413$ & $3899 \pm 3816$ \\
\hline Transport & $985 \pm 1236$ & $1094 \pm 1308$ & $410 \pm 444.22$ \\
\hline Leisure & $3226 \pm 2469$ & $3407 \pm 2428$ & $2265 \pm 2592$ \\
\hline Domestic & $1247 \pm 1429$ & $1232 \pm 1467$ & $1328 \pm 1276$ \\
\hline Sedentary & $266 \pm 127$ & $273 \pm 134$ & $232 \pm 74$ \\
\hline $\mathrm{VO}_{2} \mathrm{Max}$ & $49 \pm 5$ & $50 \pm 4$ & $43 \pm 3$ \\
\hline
\end{tabular}

Figure 1 shows descriptive statistics activity and $\mathrm{VO}_{2} \mathrm{Max}$. There is little difference in mean of physical activity between GPAQ and IPAQ. In addition, IPAQ mean total score has a significantly higher standard deviation than GPAQ mean total score.

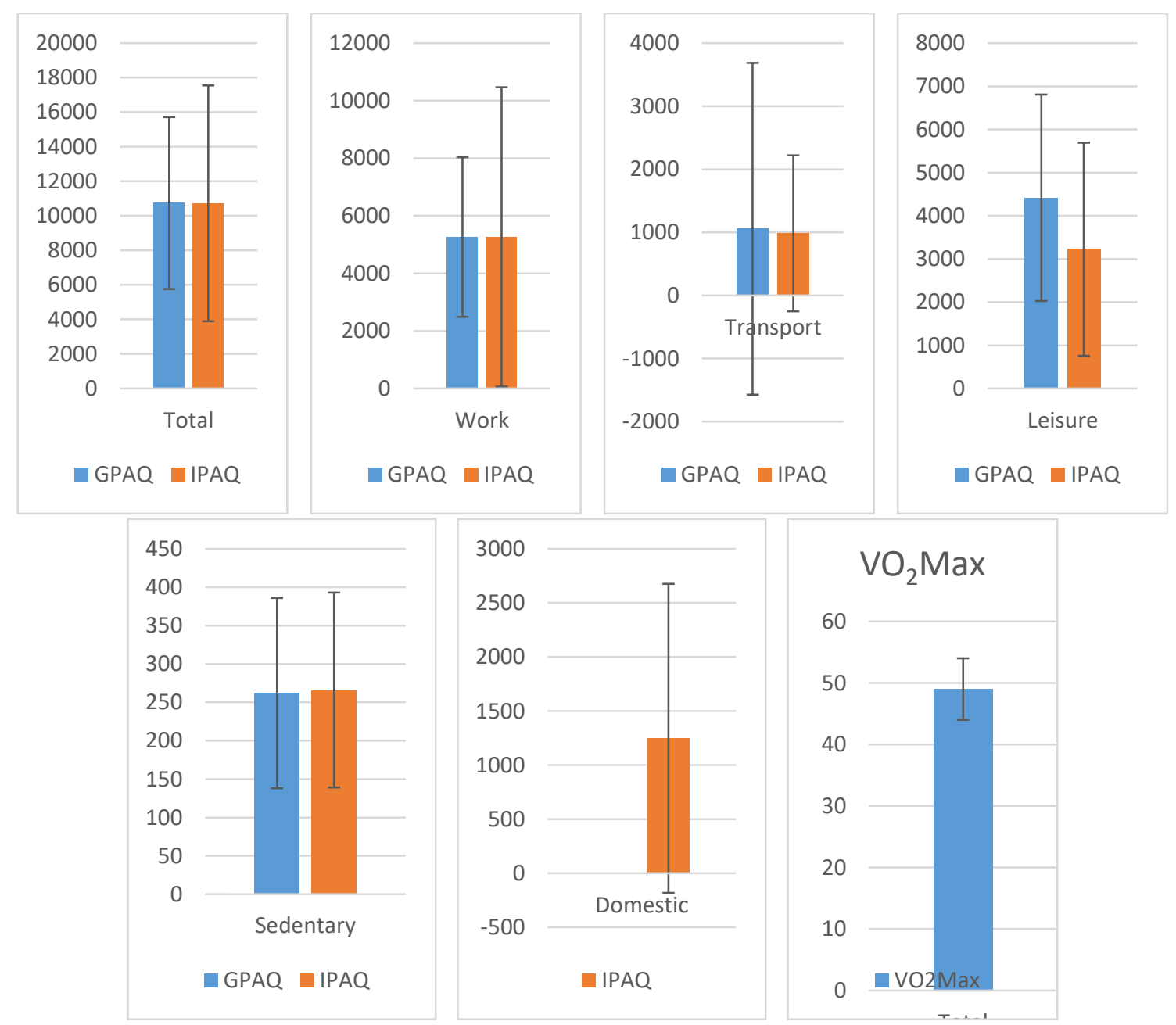

Figure 1. Mean of Physical Activity and $\mathrm{VO}_{2} \mathrm{Max}$ Total Score 
Table 3. The Comparisons of Physical Activity Measured with the IPAQ and The GPAQ (in Mets)

\begin{tabular}{|c|c|c|c|c|c|c|c|c|c|}
\hline & \multicolumn{3}{|c|}{ Total $(n=63)$} & \multicolumn{3}{|c|}{ Male $(n=53)$} & \multicolumn{3}{|c|}{ Female $(n=10)$} \\
\hline & GPAQ & IPAQ & P\# & GPAQ & IPAQ & P\# & GPAQ & IPAQ & P\# \\
\hline Total & $10737 \pm 4980$ & $10724 \pm 6826$ & $0.986 \#$ & $11242 \pm 4807$ & $11256 \pm 7104$ & $0.986 \#$ & $8058 \pm 5277$ & $7902 \pm 4342$ & $0.922 \#$ \\
\hline Work & $5262 \pm 2774$ & $5266 \pm 5201$ & 0.205 & $5485 \pm 2645$ & $5523 \pm 5413$ & 0.231 & $4080 \pm 3275$ & $3899 \pm 3816$ & 0.575 \\
\hline Transport & $1057 \pm 2630$ & $985 \pm 1236$ & $0.037 *$ & $1178 \pm 2842$ & $1094 \pm 1308$ & $0.043 *$ & $414 \pm 661$ & $410 \pm 444$ & 0.779 \\
\hline Leisure & $4418 \pm 2391$ & $3226 \pm 2469$ & $0.000 *$ & $4580 \pm 2426$ & $3407 \pm 2428$ & $0.001 \# *$ & $3564 \pm 2100$ & $2265 \pm 2592$ & $0.004 \# *$ \\
\hline Sedentary & $262 \pm 124$ & $266 \pm 127$ & 0.805 & $262 \pm 132$ & $273 \pm 134$ & 0.503 & $262 \pm 75$ & $232 \pm 74$ & 0.214 \\
\hline
\end{tabular}

\# = Total PA was measured using paired sample t-test, while the other domains calculated using Wilcoxon Signed Rank test

Table 3 shows the mean total between IPAQ and GPAQ compared using paired sample t-tests. The result was no significant difference between IPAQ and GPAQ $(p>0.05)$. Table 3 shows the mean differences of GPAQ and IPAQ domains. No significant differences were found in the work domain and sedentary domain $(p>0.05)$. Standard deviation of IPAQ work domain was greater than GPAQ work domain. However, significant differences were found in the transport domain and leisure domain $(p>0.05)$, while no significant differences were found in the transport domain in female group $(p>0.05)$. The average of transport and leisure based-related physical activity measured using GPAQ was greater than those measured using IPAQ.

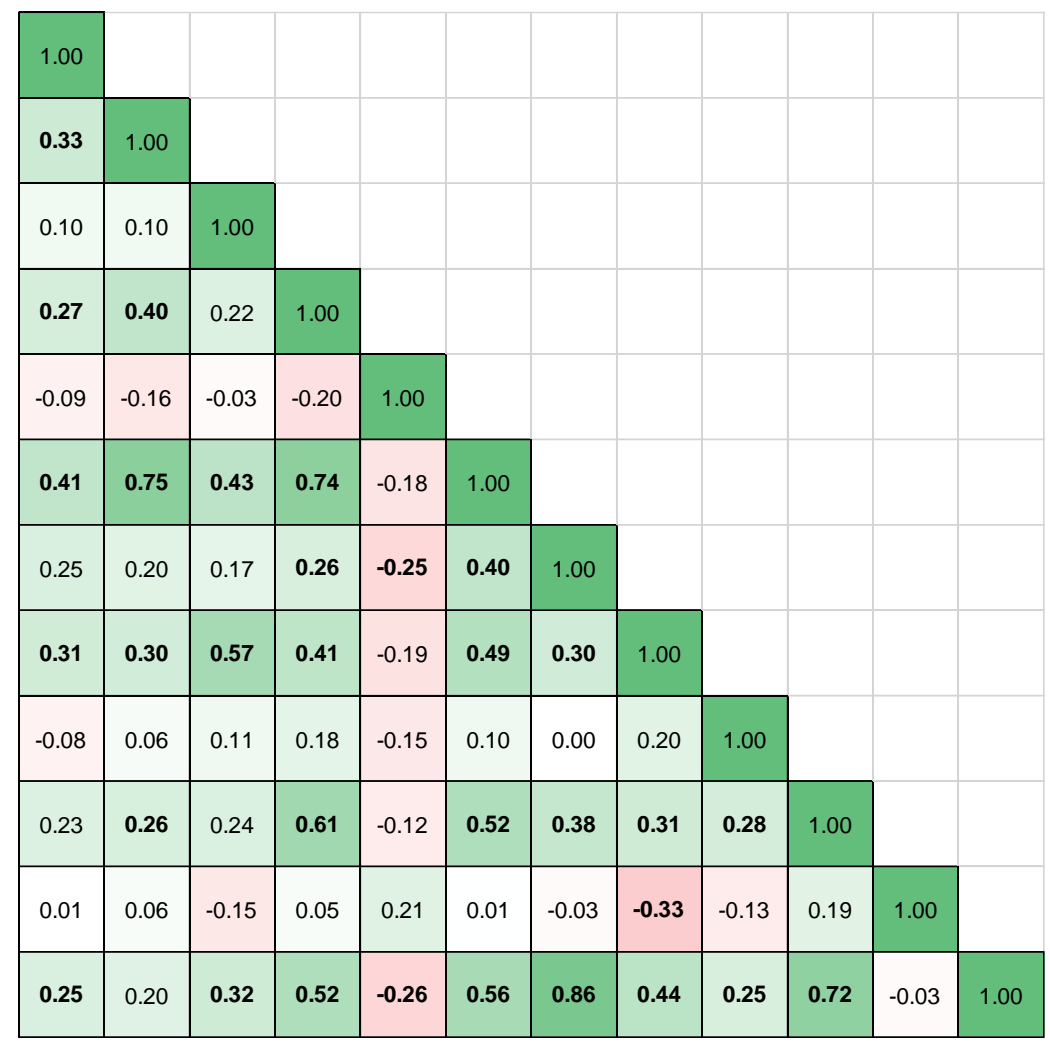

Figure 2. Correlation matrix among IPAQ, GPAQ, and $\mathrm{VO}_{2} \mathrm{Max}$

*Bold signifies a significant correlation while red and green shades signify negative and positive correlations with the darker shade represent higher correlation. 


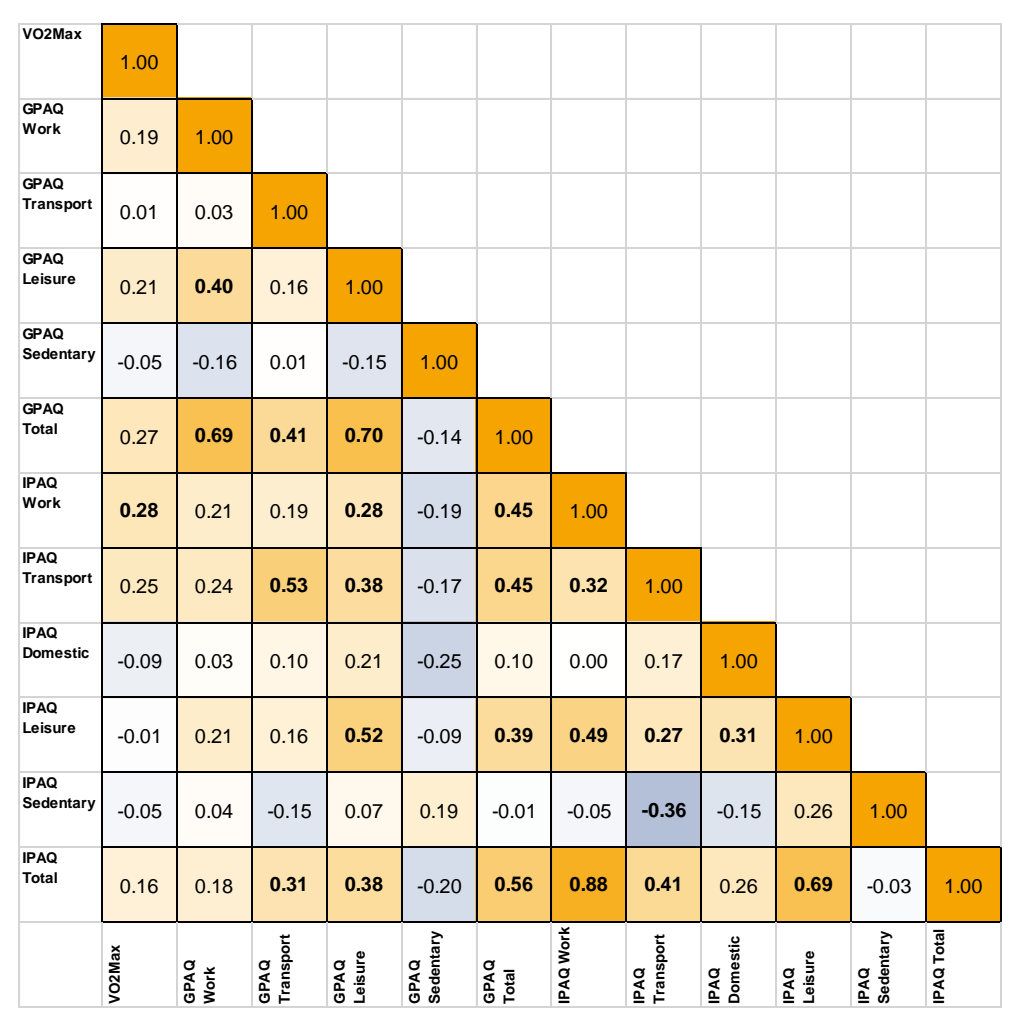

Figure 3. Correlation matrix among IPAQ, GPAQ, and $\mathrm{VO}_{2} \mathrm{Max}$ in Male

*Bold signifies a significant correlation while blue and orange shades signify negative and positive correlations with the darker shade represent higher correlation.

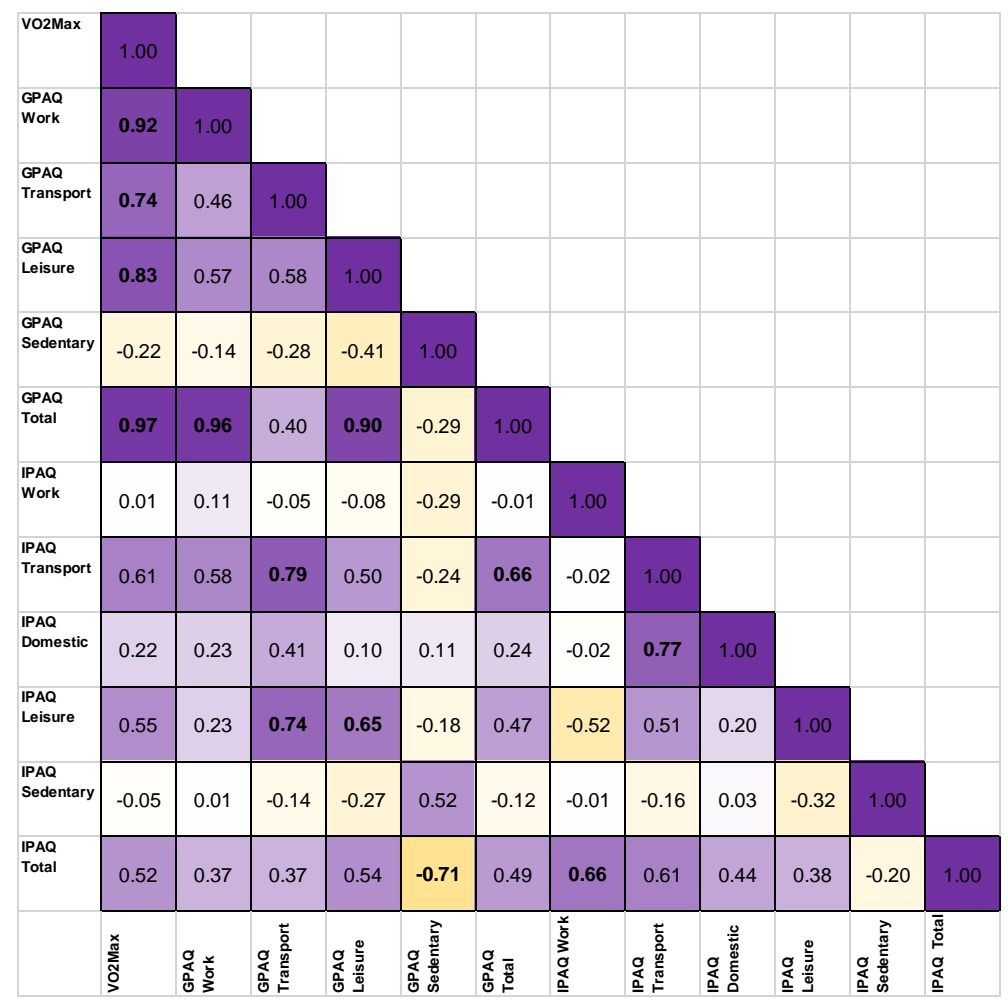

Figure 4. Correlation matrix among IPAQ, GPAQ, and $\mathrm{VO}_{2} \mathrm{Max}$ in Female

*Bold signifies a significant correlation while yellow and purple shades signify negative and positive correlations with the darker shade represent higher correlation. 
As shown in Figure 2, a moderate significant positive correlation was found between GPAQ and $\mathrm{VO}_{2} \mathrm{Max}$ (r: $0.41 ; p<0.01$ ). In this study, we found that GPAQ has a stronger correlation with $\mathrm{VO}_{2} \mathrm{Max}$ than IPAQ (GPAQ= r: 0.41, p: 0.01, IPAQ= r: 0.25; p<0.05). GPAQ also has stronger correlation with $\mathrm{VO}_{2} \mathrm{Max}$ than IPAQ in male and female group as shown in Figure 3 and Figure 4. In addition, we found that there was significant moderate positive correlation between GPAQ and IPAQ (r: $0.56 ; p: 0.01)$.

Our findings showed that men are more physically active and have higher levels of maximal oxygen capacity than women. Men's activity in sports, recreational activities, and work activities (university) also increased significantly. Female students are characterized by significantly more housework activities (Bergier, Bergier, \& Tsos, 2016). Male also had higher absolute levels of $\mathrm{VO}_{2} \mathrm{Max}$ when comparing physiological values between male and female (Koons, Suresh, Schlotman, \& Convertino, 2019).

This study did not show a significant difference between GPAQ and IPAQ. Lingesh et al., (2016) compared Malaysian version of IPAQ and GPAQ using paired sample t-test. It was found that there was no significant difference between the mean MET-min week-1 between them $(p<0.05)$. The standard deviation of the mean in IPAQ is also larger than GPAQ. It is indicating that there is a greater variance in physical activity scores using IPAQ. Study by Misra et al., (2014) indicated that GPAQ is equivalent to IPAQ for measuring physical activity and can be used to measure physical activity in community settings. Thus, it can be concluded that GPAQ can be safely used instead of IPAQ for measuring physical activity.

Although GPAQ and IPAQ did not differ in this study, they did differ in domain structure. When the GPAQ and IPAQ domains were compared, the results varied. Meanwhile, physical activity related to household chore activity (domestic domain) could not be compared because even though both instruments have similar domains, but their structures are different (Armstrong \& Bull, 2006). Work and domestic activities are presented as separate domains in IPAQ, and are formed under the work domain of GPAQ (Shaheen, Puri, \& Tandon, 2016).

Our finding was in accordance with a finding by Malavia and Shah, (2019) that explores the correlation between cardiorespiratory fitness and physical activity among the security guards aged 20 to 40 years. It was indicated in that study that there was a strong positive relationship between MET minutes of total physical activity per week and aerobic capacity (r: $0.847 ; p<0.01)$. It means that physical activity has a significant effect on physical fitness. The positive correlation coefficient indicates that the higher the physical activity score (MET), the higher the physical fitness score $\left(\mathrm{VO}_{2} \mathrm{Max}\right)$ (Kriswanto, Prasetyowati, Sunardi, \& Suharjana, 2020). However, a study by Nainggolan et al., (2018) indicated that there is no correlation between GPAQ version 2 and $\mathrm{VO}_{2} \mathrm{Max}$ using ergocycle with astrand method in women aged 25 to 54 years old (r: 0,$070 ; p: 0,4465$ ). Our study participants were majority males thus it may explain the discrepancy.

We also found that there was a weak significant positive correlation between IPAQ dan $\mathrm{VO}_{2} \mathrm{Max}$ (r: $0.25 ; p<0.05$ ). The finding is in line with the results of a study by Batista et al., (2013). They evaluated the association between muscular strength and endurance, flexibility, and cardiorespiratory in 408 healthy men aged 21-43 years with the results of IPAQ-SF. The participants performed sit-ups and push-ups, hand grip dynamometer, sit-and-reach, and 20-m shuttle run test. The study also indicated that there was a weak positive correlation between IPAQ and $\mathrm{VO}_{2} \mathrm{Max}(\mathrm{r}: 0.22 ; p$ : 0.01 ).

We also found that GPAQ has a stronger correlation with $\mathrm{VO}_{2} \mathrm{Max}$ than IPAQ and there was significant moderate positive correlation between GPAQ and IPAQ. Fernate et al., (2019) did a study to determine the correlation between the measurement attributes of the self-reported physical activity indicators in the Global Physical Activity Questionnaire (GPAQ), the International Physical Activity Questionnaire (IPAQ), and the European Health Survey Physical Activity Questionnaire (EHISPAQ) for 70 gym clients aged 18 to 70 years, and evaluate them simultaneously effective. The result of the study was all three questionnaires was significantly correlated. It was also found that there are five close correlations between GPAQ and IPAQ content indicators $(p<0.05)$ thus support the results of our study. The stronger correlation between GPAQ and $\mathrm{VO}_{2} \mathrm{Max}$ compared to IPAQ and $\mathrm{VO}_{2} \mathrm{Max}$ may also indicate that the household-related item in the IPAQ may not be neceserally relevant to young adults as they may not be yet responsible to household chores. Based on the finding, we recommend the use of the GPAQ over the use of IPAQ in this population. 
To the best of our knowledge, our study is the first to ascertain the convergent validity between IPAQ and GPAQ in young adults who were predominantly active. We however acknowledge several limitations. First this study only based on a relatively small sample size thus may need to be confirmed with study with larger sample size. Another limitation is due to the use of self-report questionnaires. Some participants may have intentionally or unintentionally misreported. Future research is recommended to conduct with larger sample and various populations.

\section{CONCLUSION}

The present study indicates convergent validity between the GPAQ and IPAQ thus justify the use of the instruments in young adults population in Indonesia. We also found that GPAQ has a higher correlation with $\mathrm{VO}_{2} \mathrm{Max}$ compared to the IPAQ and $\mathrm{VO}_{2} \mathrm{Max}$. We, therefore, recommend the use of the GPAQ compare to the IPAQ to measure physical activity in this population.

\section{ACKNOWLEDGMENT}

The authors would like to thank all students of Physical, Health, and Recreation Education study program at Sports Science Faculty, Yogyakarta State University for their support as participants in this study.

\section{CONFLICT OF INTEREST}

The authors declare that they have no competing interests in this study. All authors have been read and approved the manuscript for submission.

\section{REFERENCES}

Ács, P., Betlehem, J., Oláh, A., Bergier, B., Morvay-Sey, K., Makai, A., \& Prémusz, V. (2020). Crosscultural adaptation and validation of the Global Physical Activity Questionnaire among healthy Hungarian adults. BMC Public Health, 20(1), 1-10. https://doi.org/10.1186/s12889-020-08477-z

Armstrong, T., \& Bull, F. (2006). Development of the World Health Organization Global Physical Activity Questionnaire ( GPAQ ). Journal of Public Health, 14(2), 66-70. https://doi.org/10.1007/s10389-0060024-X

Bahnemiri, Z. (40). risr. J., Roshan, V. D., \& Movaghar, A. F. (2018). Non Invasive Estimation of Vo2max and Physical Fitness Indices in Mazandaran University of Medical Sciences Staff. Journal of Mazandaran University of Medical Sciences, 28(159), 74-83.

Batista, C. S., Urso, Ro. P., Silva, Ad. E. Li., \& Bertuzzi, R. (2013). Associations Between Fitness Tests And The International Physical Activity Questionnaire-Short Form In Healthy Men. Journal of Strength and Conditioning Research, 27(12), 3481-3487.

Bergier, J., Bergier, B., \& Tsos, A. (2016). Variations in Physical Activity of Male and Female Students from Different Countries. Iran J Public Health, 45(5), 705-707.

Bull, F. C., Al-Ansari, S. S., Biddle, S., Borodulin, K., Buman, M. P., Cardon, G., ... Willumsen, J. F. (2020). World Health Organization 2020 guidelines on physical activity and sedentary behaviour. British Journal of Sports Medicine, 54(24), 1451-1462. https://doi.org/10.1136/bjsports-2020-102955

Cain, K. L., Sallis, J. F., Conway, T. L., Van Dyck, D., \& Calhoon, L. (2013). Using accelerometers in youth physical activity studies: a review of methods. Journal of Physical Activity \& Health, 10(3), 437450. https://doi.org/10.1123/jpah.10.3.437

Craig, C. L., Marshall, A. L., Sjöström, M., Bauman, A. E., Booth, M. L., Ainsworth, B. E., ... Oja, P. (2003). International physical activity questionnaire: 12-Country reliability and validity. Medicine and Science in Sports and Exercise, 35(8), 1381-1395. https://doi.org/10.1249/01.MSS.0000078924.61453.FB 
Cureton, K. J., Sloniger, M. A., O’Bannon, J. P., Black, D. M., \& McCormack, W. P. (1995). A generalized equation for prediction of VO2peak from 1-mile run/walk performance. Medicine and Science in Sports and Exercise, Vol. 27, pp. 445-451. https://doi.org/10.1249/00005768-199503000-00023

Fernate, A., Vazne, Z., \& Levskojs, A. (2019). Analysis of the Self-Reported Physical Activity Questionnaires Indicators. Society. Integration. Education. Proceedings of the International Scientific Conference, 4, 144-153. https://doi.org/10.17770/sie2019vol4.3746

Hallal, P. C., Gomez, L. F., Parra, D. C., Lobelo, F., Mosquera, J., Florindo, A. A., ... Sarmiento, O. L. (2010). Lessons learned after 10 years of IPAQ use in Brazil and Colombia. Journal of Physical Activity and Health, 7(2), 259-264. https://doi.org/10.1123/jpah.7.s2.s259

Haskell, W. L., Lee, I. M., Pate, R. R., Powell, K. E., Blair, S. N., Franklin, B. A., .. Bauman, A. (2007). Physical activity and public health: Updated recommendation for adults from the American College of Sports Medicine and the American Heart Association. Medicine and Science in Sports and Exercise, 39(8), 1423-1434. https://doi.org/10.1249/mss.0b013e3180616b27

Herrmann, S. D., Heumann, K. J., Der Ananian, C. A., \& Ainsworth, B. E. (2013). Validity and reliability of the global physical activity questionnaire (GPAQ). Measurement in Physical Education and Exercise Science, 17(3), 221-235. https://doi.org/10.1080/1091367X.2013.805139

IPAQ Research Committee. (2005). Guidelines for data processing and analysis of the International Physical Activity Questionnaire (IPAQ)—Short and Long Forms.

Kamyan, D., Labania, L., Kamyan, A., Rahman, M., \& Bagchi, S. (2020). Assessment of Cardiorespiratory Endurance in Terms of Physical Fitness Index and VO2max among Young adult population of United Arab Emirates. International Medical Journal (1994), 25(04), 1927-1940.

Koons, N. J., Suresh, M. R., Schlotman, T. E., \& Convertino, V. A. (2019). Interrelationship between sex, age, blood volume, and V'o 2max. Aerospace Medicine and Human Performance, 90(4), 362-368. https://doi.org/10.3357/AMHP.5255.2019

Kriswanto, E. S., Prasetyowati, I., Sunardi, J., \& Suharjana, F. (2020). The Influence of Quality of Sleep and Physical Activity on Physical Fitness. Proceedings Ofthe 3rdYogyakarta International Seminar on Health, Physical Education, and Sport Science in Conjunction with the 2nd Conference on Interdisciplinary Approach in Sports (YISHPESS and CoIS 2019), 465-470. https://doi.org/10.5220/0009788804650470

Lingesh, G., Khoo, S., Nahar, M., Mohamed, A., Taib, N. A., \& Group, M. (2016). Comparing physical activity levels of Malay version of the IPAQ and GPAQ with accelerometer in nurses. International Journal of Applied Exercise Physiology, 5(3), 8-17.

Malavia, B., \& Shah, S. (2019). Association Between the Levels of Physical Activity and Cardiorespiratory Fitness Among Security Guards. International Journal of Physiotherapy and Research, 7(2), 30163021. https://doi.org/10.16965/ijpr.2019.103

Misra, P., Upadhyay, R. P., Krishnan, A., Sharma, N., \& Kapoor, S. K. (2014). A community based study to test the reliability and validity of physical activity measurement techniques. International Journal of Preventive Medicine, 5(8), 952.

Nainggolan, O., Indrawati, L., \& Pradono, J. (2018). Physical Fitness According to GPAQ Instruments Compared with Vo2Max in Women Age 25 to 54 Years Old. Puslitbang Upaya Kesehatan Masyarakat - Badan Litbangkes - Kemenkes RI, 21(4), 271-280.

Piccinno, A., \& Colella, D. (2017). Differences in cardiovascular fitness of Italian high-school adolescents according to different physical activity levels assessed by IPAQ-A: a cross-sectional study. Sport Sciences for Health, 13(1), 149-155. https://doi.org/10.1007/s11332-016-0326-5 
Raustorp, A., \& Fröberg, A. (2018). Tracking of pedometer-determined physical activity: A 16-Year followup study. Journal of Physical Activity and Health, 15(1), 7-12. https://doi.org/10.1123/jpah.2017-0146

Rhodes, R. E., Janssen, I., Bredin, S. S. D., Warburton, D. E. R., \& Bauman, A. (2017). Physical activity: Health impact, prevalence, correlates and interventions. Psychology and Health, 32(8), 942-975. https://doi.org/10.1080/08870446.2017.1325486

Shaheen, M., Puri, S., \& Tandon, N. (2016). Physical Activity Measurement in Elderly: The Indian Scenario. Journal of Physical Activity Research, 1(1), 9-14. https://doi.org/10.12691/jpar-1-1-3

Soo, K. L., Wan Abdul Manan, W. M., \& Wan Suriati, W. N. (2015). The bahasa melayu version of the global physical activity questionnaire: Reliability and validity study in Malaysia. Asia-Pacific Journal of Public Health, 27(2), NP184-NP193. https://doi.org/10.1177/1010539511433462

Stelmach, M. (2018). Physical activity assessment tools in monitoring physical activity: the Global Physical Activity Questionnaire (GPAQ), the International Physical Activity Questionnaire (IPAQ) or accelerometers - choosing the best tools. Health Problems of Civilization, 12(1), 57-63. https://doi.org/10.5114/hpc.2018.74189

Taylor, P., Tomaz, S. A., Lambert, E. V, \& Karpul, D. (2016). Cardiovascular fitness is associated with bias between self-reported and objectively measured physical activity. European Journal of Sport Science, 16(1), 149-157. https://doi.org/10.1080/17461391.2014.987323

Wanner, M., Hartmann, C., Pestoni, G., Martin, B. W., Siegrist, M., \& Martin-Diener, E. (2017). Validation of the Global Physical Activity Questionnaire for self-administration in a European context. BMJ Open Sport and Exercise Medicine, 3(1), 1-9. https://doi.org/10.1136/bmjsem-2016-000206

WHO. (2012). Global Physical Activity Questionnaire (GPAQ) Analysis Guide. Retrieved November 15, 2021, from https://www.who.int/ncds/surveillance/steps/resources/GPAQ_Analysis_Guide.pdf 